Pacific Journal of Mathematics

CHARACTERIZING REDUCED WITT RINGS OF HIGHER 


\title{
CHARACTERIZING REDUCED WITT RINGS OF HIGHER LEVEL
}

\author{
VICTORIA POWERS
}

\begin{abstract}
Mulcahy's Spaces of Signatures (SOS) is an abstract setting for the reduced Witt rings of higher level of Becker and Rosenberg just as Marshall's Spaces of Orderings is an abstract setting for the ordinary reduced Witt ring. Finitely constructible SOS's are those built up in a finite number of steps from the smallest SOS using 2 operations. We show that finitely constructible SOS's are precisely those that arise from preordered fields (subject to a certain finiteness condition). This allows us to give an inductive construction for the reduced Witt rings of higher level for certain preordered fields, which generalizes a result of Craven for the ordinary reduced Witt ring. We also obtain a generalization of Bröcker's results on the possible number of orderings of a field.
\end{abstract}

1. Preliminaries. For a field $K$ we set $\dot{K}=K \backslash\{0\}$. The symbol $\sqcup$ stands for disjoint union. We begin by recalling some of the theory of preorders of higher level from [4]:

A subset $T$ of $K$ is a preorder if $\dot{T}=T \backslash\{0\}$ is a subgroup of $\dot{K}$ and $\dot{T}+\dot{T} \subseteq \dot{T}$. We assume throughout that all preorders are of finite exponent, i.e., $K^{m} \subseteq T$ for some $m \in \mathbf{N}$. Since $-1 \notin \dot{T}$, the exponent of the group $\dot{K} / \dot{T}$ is even, say $2 n$. We call $n$ the level of $T$.

Let $\mu=\left\{z \in \mathbf{C}: z^{r}=1\right.$ for some $\left.r \in \mathbf{N}\right\}$. For an abelian group $G$ let $G^{*}$ denote $\operatorname{Hom}(G, \mu)$, with the usual compact-open topology. $\chi \in(\dot{K})^{*}$ is called a signature if $\operatorname{ker} \chi+\operatorname{ker} \chi \subseteq \operatorname{ker} \chi$. We write $\operatorname{Sgn}(K)$ for the set of signatures of $K$. For a preorder $T$ let $X_{T}=\{\chi \in \operatorname{Sgn}(K): \chi(\dot{T})=1\}$. Then $\dot{T}=\bigcap_{\chi \in X_{T}} \operatorname{ker} \chi[4,1.4]$.

We make extensive use of Krull valuations: If $v: \dot{K} \rightarrow \Gamma$ is a valuation, we denote the valuation ring by $A$, the group of units by $U$, the maximal ideal by $I$ and the residue class field by $k$. If $k$ is formally real, we say $v$ is a real valuation.

An element $\chi \in(\dot{K})^{*}$ is "compatible" with a valuation $v$, written $v \sim \chi$, if $1+I \subseteq \operatorname{ker} \chi$. In this case, the equation $\bar{\chi}(u+I)=\chi(u)$ defines an element $\bar{\chi} \in(\dot{k})^{*}$, called the pushdown of $\chi$ along $v$, and $\chi \in \operatorname{Sgn}(K)$ iff $\bar{\chi} \in \operatorname{Sgn}(k)[3,1.12,2.5]$. A preorder $T$ of $K$ is "fully compatible", written $v \sim{ }_{f} T$, if each $\chi \in X_{T}$ is compatible with $v$, i.e., if $1+I \subseteq T$. In that case the image of $A \cap T$ in $k$, denoted $\bar{T}$, is a preorder of $k$. 
For $\chi \in \operatorname{Sgn}(K)$, let $A(\chi)=\{a \in K$ : there is an $n \in \mathbf{N}$ with $n+a$ and $n-a \in \operatorname{ker} \chi\}$. Then $A(\chi)$ is the smallest valuation ring such that $A(\chi) \sim \chi$ and the pushdown of $\chi$ along $A(\chi)$ is archimedean $[3,2.7]$.

Definition 1.1 (cf. [13], [17]). Let $G$ be an abelian group of exponent $2 s$, for some $s \in \mathbf{N}$. Let $X \subseteq G^{*}$ be nonempty. An $n$-dimensional form $f$ over $(X, G)$ is an $n$-tuple $\left\langle a_{1}, \ldots, a_{n}\right\rangle$ with $a_{i} \in G$. We write $\operatorname{dim} f=n$ and, for $\sigma \in X, \sigma(f)=\sum_{i=1}^{n} \sigma\left(a_{i}\right)$. Two forms $f$ and $g$ are said to be equivalent if $\sigma(f)=\sigma(g)$ for all $\sigma \in X$. We write $f \equiv g$. $f$ and $g$ are isometric, written $f \cong g$, if $f \equiv g$ and $\operatorname{dim} f=\operatorname{dim} g$. We say a form $f$ represents $x \in G$ if $f \cong\left\langle x, x_{2}, \ldots, x_{n}\right\rangle$ for some $x_{i} \in G$. $D(f)$ denotes the set of all elements of $G$ represented by $f$. The sum, $f \oplus g$, and the product, $f \otimes g$, are defined in the usual way (see [13]) and the form $\langle a\rangle \otimes f, a \in G$, is denoted af. We use the convention that there is an empty form \langle\rangle , i.e., a form with no entries. $\operatorname{Dim}(\langle\rangle)=0$ and $\sigma(\langle\rangle)=0$.

The pair $(X, G)$ is called a Space of Signatures, or SOS, when the following axioms hold:

$S_{0}$ : For any $\sigma \in X$ and any odd integer $k, \sigma^{k} \in X$.

$S_{1}: X$ is closed in $G^{*}$.

$S_{2}$ : There is an $e \in G$ such that $\sigma(e)=-1$ for all $\sigma \in X$.

$S_{3}: X^{\perp}=\{a \in G: \sigma(a)=1$ for all $\sigma \in X\}=1$.

$S_{4}$ : If $f$ and $g$ are forms over $(X, G)$ and $z \in D(f \oplus g)$, then there is an $x \in D(f)$ and a $y \in D(g)$ such that $z \in D(\langle x, y\rangle)$.

$S_{5}$ : If $\sigma \in G^{*} \backslash\{1\}$ is such that $D(\langle 1, x\rangle) \subseteq \operatorname{ker} \sigma$, for all $x \in \operatorname{ker} \sigma$, then $\sigma \in X$.

\section{REMARKS 1.2.}

(i) By $S_{3}$, the $e$ of $S_{2}$ is unique and we denote it by -1 .

(ii) If $G^{2}=1$, then a Space of Signatures is precisely what Marshall called a Space of Orderings $[13,14,15,16]$.

(iii) If $T$ is a preorder of $K$, then $\left(X_{T}, \dot{K} / \dot{T}\right)$ is an SOS [17, 1.10(iii)].

(iv) If $\left(X_{1}, G_{1}\right)$ and $\left(X_{2}, G_{2}\right)$ are two SOS's and there is an isomorphism $\alpha: G_{1} \rightarrow G_{2}$ such that $\alpha^{*}\left(X_{2}\right)=X_{1}$, where $\alpha^{*}$ denotes the dual map, we will write $\left(X_{1}, G_{1}\right)=\left(X_{2}, G_{2}\right)$.

Definition 1.3. An $\operatorname{SOS}(X, G)$ is realizable if there exists a field $K$ and a preorder $T$ such that $\left(X_{T}, \dot{K} / \dot{T}\right)=(X, G)$.

EXAMPLES 1.4. (i) Let $G=\{ \pm 1\}$ be the 2-element group. Let $X \subseteq G^{*}$ consist of the character that sends -1 to -1 . We denote the pair $(X, G)$ by $\mathscr{C}_{2}$. Obviously $\mathscr{C}_{2}$ is realizable, for example by $K=\mathbf{R}$ and $T=\mathbf{R}^{2}$. 
(ii) (cf. $[17,1.10(i i i)])$. Let $G$ be any group of finite even exponent, and fix an element -1 of order 2. Set $X=\left\{\chi \in G^{*}: \chi(-1)=-1\right\}$. By analogy with $[4$, p. 448$]$ we call $(X, G)$ a fan. We will see later that all fans are realizable SOS's.

2. Group extensions and direct sums. In this section we examine two ways in which a SOS can be built up out of "smaller" SOS's. Our main goal is to show that if we start with realizable SOS's our new SOS is also realizable.

Definition 2.1 (cf. $[15,3.6])$. Suppose $\left(X_{0}, G_{0}\right)$ is a SOS and $G$ is an abelian group of finite exponent with $G_{0} \subset G$. Set $X=\left\{\chi \in G^{*}:\left.\chi\right|_{G_{0}} \in\right.$ $\left.X_{0}\right\}$. The pair $(X, G)$ is called a group extension of $\left(X_{0}, G_{0}\right)$. We shall say that $(X, G)$ is a group extension if there exists $\left(X_{0}, G_{0}\right)$ such that $(X, G)$ is a group extension of $\left(X_{0}, G_{0}\right)$. If $(X, G)$ is a group extension, then it is a SOS with $-1_{G}=-1_{G_{0}}[17,2.6]$.

REMARKS 2.2. (i) Group extensions of a given SOS $\left(X_{0}, G_{0}\right)$ correspond to abelian group extensions of $G_{0}$ : If $(X, G)$ is a group extension of $\left(X_{0}, G_{0}\right)$ then we have an exact sequence of abelian groups $1 \rightarrow G_{0} \rightarrow G$ $\rightarrow G / G_{0} \rightarrow 1$. Conversely, given an exact sequence $1 \rightarrow G_{0} \rightarrow G \rightarrow H \rightarrow$ 1 where $H$ is abelian of finite exponent, then setting $X=\left\{\chi \in G^{*}\right.$ : $\left.\left.\chi\right|_{G_{0}} \in X_{0}\right\}$ and identifying $G_{0}$ with its image in $G$, we see that the pair $(X, G)$ is a group extension of $\left(X_{0}, G_{0}\right)$.

(ii) Suppose $(X, G)$ is a group extension of $\left(X_{0}, G_{0}\right)$. Since $\mu$ is divisible, it is $\mathbf{Z}$-injective [7, Prop. 5.1, p. 134] and thus there is a dual exact sequence

$$
1 \rightarrow\left(G / G_{0}\right)^{*} \rightarrow G^{*} \stackrel{\text { res }}{\rightarrow} G_{0}^{*} \rightarrow 1,
$$

where res is the restriction map. Then $\chi \in X$ iff $\operatorname{res}(\chi) \in X_{0}$, and thus there is a non-canonical bijection $X \leftrightarrow X_{0} \times\left(G / G_{0}\right)^{*}$.

(iii) Clearly $(X, G)$ is a fan iff $(X, G)$ is a group extension of $\mathscr{C}_{2}$ or $\mathscr{C}_{2}$ itself.

(iv) Equivalent abelian group extensions, in the sense of [10, p. 211], give rise to the "same" SOS, in the sense of $[1.2,(\mathrm{v})]$.

Proposition 2.3. Let $K$ be a field, $T \subseteq K$ a preorder and $v$ a valuation such that $v \sim{ }_{f} T$. If $\Gamma / v(\dot{T})=1$, then $\left(X_{T}, \dot{K} / \dot{T}\right)=\left(X_{\bar{T}}, \dot{k} / \dot{\bar{T}}\right)$. If $\Gamma / v(\dot{T}) \neq 1$, then $\left(X_{T}, \dot{K} / \dot{T}\right)$ is a group extension of $\left(X_{\bar{T}}, \dot{k} / \dot{\bar{T}}\right)$. 
Proof. By $[3,2.6]$ the sequence

$$
1 \rightarrow \dot{k} / \dot{\bar{T}} \stackrel{\alpha}{\rightarrow} \dot{K} / \dot{T} \stackrel{\beta}{\rightarrow} \Gamma / v(\dot{T}) \rightarrow 1
$$

is exact, where $\alpha(\overline{u T})=u \dot{T}$ and $\beta(a \dot{T})=v(a) v(\dot{T})$. By $[4,2.7] \alpha^{*}(\chi) \in$ $X_{\bar{T}}$ iff $\chi \in X_{T}$.

COROLlaRY 2.4. Given a field $K$, a preorder $T$ and $a$ valuation $v$ such that $v \sim{ }_{f} T$. Then $T$ is a fan iff $\bar{T}$ is a fan.

Proof. This follows easily from [2.3] if we note the following: If $(X, G)$ is a group extension of $\left(X_{0}, G_{0}\right)$, then for any $\chi \in G^{*}, \chi(-1)=-1$ iff $\left.\chi\right|_{G_{0}}(-1)=-1$.

Now we fix a field $F$, a preorder $T \subseteq F$ and a $\operatorname{SOS}(X, G)$ which is a group extension of $\left(X_{T}, \dot{F} / \dot{T}\right)$. Thus we have an exact sequence

$$
1 \rightarrow \dot{F} / \dot{T} \rightarrow G \rightarrow H \rightarrow 1 \text {. }
$$

We want to show that $(X, G)$ is realizable. To do this we will construct a field $K$, a preorder $Q \subseteq K$, and a valuation $v \sim_{f} Q$ such that the exact sequence of [2.3] is equivalent to (1).

Proposition 2.5. (With the above notation), $(X, G)$ is realizable.

Proof. Fix $\lambda^{\prime}: H \times H \rightarrow \dot{F} / \dot{T}$, a factor set corresponding to the exact sequence (1). Since $H$ is abelian of finite exponent, $H \cong \Gamma / \Delta$ where $\Gamma$ is a direct sum of copies of $Z[10,15.2]$. Order $\Gamma$ lexicographically. By [10, 51.3], there is a factor set $\lambda: \Gamma \times \Gamma \rightarrow F$ such that $\lambda\left(\gamma_{1}, \gamma_{1}\right) \dot{T}=$ $\lambda^{\prime}\left(\gamma_{1} \Delta, \gamma_{2} \Delta\right)$ for all $\gamma_{1}, \gamma_{2} \in \Gamma$.

Let $K$ be the formal power series of $\Gamma$ over $F$ : This is the set of functions $\Omega: \Gamma \rightarrow \dot{F}$ with $S(\Omega)$, the support of $\Omega$, well-ordered. Addition is defined in the obvious way and multiplication is given by

$$
\left(\Omega_{1} \Omega_{2}\right)(\gamma)=\sum_{\gamma_{1} \gamma_{2}=\gamma} \lambda\left(\gamma_{1}, \gamma_{2}\right) \Omega_{1}\left(\gamma_{1}\right) \Omega_{2}\left(\gamma_{2}\right)
$$

for all $\Omega_{i} \in K$ and $\gamma \in \Gamma$. Then $K$ is a field and the map $v: K \rightarrow \Gamma$, defined by $v(\Omega)=$ least element of $S(\Omega)$, is a valuation with value group $\Gamma$ and residue class field $F[20,5.3$ and 5.4].

Let $Q=\{\Omega \in K: v(\Omega) \in \Delta$ and $\Omega(v(\Omega)) \in T\}$. It is straightforward to check that $Q$ is a preorder, $v \sim{ }_{f} Q, \bar{Q}=T$, and $v(\dot{Q})=\Delta$.

We want to show that $\dot{K} / \dot{Q} \cong G$. Since $\lambda^{\prime}$ is a factor set corresponding to (1), we can identify $G$ with pairs $(a \dot{T}, h), a \in \dot{F}$ and $h \in H$, where

$$
\left(a_{1} \dot{T}, h_{1}\right)\left(a_{2} \dot{T}, h_{2}\right)=\left(\lambda^{\prime}\left(h_{1}, h_{2}\right) a_{1} a_{2} \dot{T}, h_{1} h_{2}\right) \text {. }
$$


Define $\theta: \dot{K} \rightarrow G$ via $\theta(\Omega)=(\Omega(v(\Omega)) \dot{T}, v(\Omega) \Delta)$. Since the "twisting" in $K$ is via $\lambda$ and that of $G$ is via $\lambda^{\prime}$, a straightforward check shows that $\theta$ is a homomorphism. It is clear that $\theta$ is surjective and $\operatorname{ker} \theta=\dot{Q}$. Hence $\dot{K} / \dot{Q} \cong G$.

By [2.3], $\left(X_{Q}, \dot{K} / \dot{Q}\right)$ is the group extension of $\left(X_{T}, \dot{F} / \dot{T}\right)$ arising from the exact sequence

$$
1 \rightarrow \dot{F} / \dot{T} \rightarrow \dot{K} / \dot{Q} \stackrel{\bar{v}}{\rightarrow} H \rightarrow 1
$$

where $\bar{v}(\Omega+\dot{Q})=v(\Omega)+\Delta$. An easy check shows that (1) and (2) are equivalent exact sequences and thus $(X, G)=\left(X_{Q}, \dot{K} / \dot{Q}\right)$.

REMARK 2.6. For $G^{2}=1$ and $T=\sum F^{2}[2.5]$ is easy (see [8, 2.4]). In this case the field $K$ is an iterated power series field $F\left(\left(t_{1}\right)\right)\left(\left(t_{2}\right)\right) \ldots$, where the number of variables is equal to $\operatorname{dim} F_{2}(H)$.

Definition 2.7. Let $\left(X_{1}, G_{1}\right)$ and $\left(X_{2}, G_{2}\right)$ be two SOS's and set $G=G_{1} \times G_{2}$. Then $G^{*} \cong G_{1}^{*} \times G_{2}^{*}$ and thus $X_{1} \times\{1\}$ and $\{1\} \times X_{2}$ embed in $G^{*}$. Set $\left.X=\left(X_{1} \times\{1\}\right) \cup(\{1\}) \times X_{2}\right)$. This union is clearly disjoint and thus we write $X=X_{1} \sqcup X_{2}$. The pair $(X, G)$ is a SOS [17, 2.3], called the direct sum of $\left(X_{1}, G_{1}\right)$ and $\left(X_{2}, G_{2}\right)$ and we write $(X, G)=$ $\left(X_{1}, G_{1}\right) \oplus\left(X_{2}, G_{2}\right)$.

THEOREM 2.8. Suppose $K_{1}$ and $K_{2}$ are fields with preorders $T_{i} \subseteq K_{i}$. Then $\left(X_{T_{1}}, \dot{K}_{1} / \dot{T}_{1}\right) \oplus\left(X_{T_{2}}, \dot{K}_{2} / \dot{T}_{2}\right)$ is realizable.

The case of $T_{i}=\sum K_{i}^{2}$ is proven (in a different form) by Craven [8, 2.4]. The proof uses a construction of Bröcker [6] (see also [19, §1]). To prove [2.8] we need to construct a field $F$ and a preorder $T$ such that $\dot{F} / \dot{T} \cong \dot{K}_{1} / \dot{T}_{1} \times \dot{K}_{2} / \dot{T}_{2}$ and $X_{T}=X_{T_{1}} \sqcup X_{T_{2}}$. The construction of $F$ and $T$ will follow along the lines of Bröcker's construction. However, we will need new methods to show that $X_{T}=X_{T_{1}} \sqcup X_{T_{2}}$ : We make use of an equivalence relation on $X_{T}$ defined in [4]. Before proving [2.8], we need several lemmas.

LEMMA 2.9 (cf. [19, 1.1]). Let $K$ be a field and $v_{1}, \ldots, v_{r}$ pairwise independent (non-trivial) valuations on $K$. Suppose for each $i, 1 \leq i \leq r$, we have $\left(K_{i}, v_{i}^{\prime}\right)$ an immediate extension of $\left(K, v_{i}\right)[9, p .24]$ together with a preorder $T_{i}$ of $K_{i}$ such that $v_{i}^{\prime} \sim{ }_{f} T_{i}$. Then the diagonal map $\dot{K} \rightarrow \dot{K}_{1} / \dot{T}_{1}$ $\times \cdots \times \dot{K}_{r} / \dot{T}_{r}$ is onto. 
Proof. Let $y$ in $\dot{K}_{1} / \dot{T}_{1} \times \cdots \times \dot{K}_{r} / \dot{T}_{r}$ be $\left(m_{1} \dot{T}_{1}, \ldots, m_{r} \dot{T}_{r}\right)$. Since $\left(K_{i}, v_{i}^{\prime}\right)$ is an immediate extension of $\left(K, v_{i}\right)$, for each $i$ there is an $a_{i} \in \dot{K}$ such that $v_{i}\left(a_{i}\right)=v_{i}^{\prime}\left(m_{i}\right)$, thus $m_{i} / a_{i}=u_{i}^{\prime}$ for some unit $u_{i}^{\prime}$ in $K_{i}$. Then there is a unit $u_{i}$ in $\left(K, v_{i}\right)$ such that $u_{i}^{\prime}+I_{i}^{\prime}=u_{i}+I_{i}$. Hence $m_{i}=$ $\left(a_{i} u_{i}\right)\left(1+x_{i}\right)$ for some $x_{i} \in I_{i}^{\prime}$.

Let $n=\max \left\{\right.$ level $\left.T_{i}\right\}$. By the Approximation Theorem for Independent Valuations [5, Chap. 6, §7, No. 2, Thm. 1], there is an $a \in K$ such that $v_{i}\left(a-\left(a_{i} u_{i}\right)^{2 n-1}\right)>v_{i}\left(\left(a_{i} u_{i}\right)^{2 n-1}\right)$, hence $a=\left(a_{i} u_{i}\right)^{2 n-1}\left(1+y_{i}\right)$ for some $y_{i} \in I_{i}$. Thus we have $a m_{i}=\left(a_{i} u_{i}\right)^{2 n}\left(1+x_{i}\right)\left(1+y_{i}\right)$ and hence $a m_{i} \in T_{i}$. Thus $1 / a$ maps to $y$ and we are done.

LEMMA 2.10. Given a field $K$ and preorder $T$ of level $\leq n$. Suppose $v$ is a valuation on $K$ such that the value group $\Gamma$ is $2 n$-divisible and $v \sim{ }_{f} T$. Then $\left(X_{T}, \dot{K} / \dot{T}\right)=\left(X_{\bar{T}}, \dot{k} / \dot{\bar{T}}\right)$.

Proof. An easy generalization of [12, 3.7] shows that $v\left(\sum K^{2 n}\right)=2 n \Gamma$. Thus $2 n \Gamma \subseteq v(\dot{T})$ and hence $\Gamma / v(\dot{T})=1$. The lemma now follows from [2.3].

LEMMA 2.11. Let $v$ be a henselian valuation on $K$ with residue class field $k$. Then any preorder of $K$ is fully compatible with $v$ and given any preorder $Q$ of $k$ there is a preorder $T$ of $K$ such that $\bar{T}=Q$.

Proof. An easy generalization of $[12,4.16]$ shows that a henselian valuation is fully compatible with $\sum K^{2 n}$ for any $n$, and hence with any preorder. Let $S=\sum K^{2 n}$, where $n=$ level $Q$, then clearly $\bar{S} \subseteq Q$. We let $T$ be the "wedge product" $S \wedge Q=S \cdot\{u \in U: \bar{u} \in Q\}$. By the first statement, $v \sim{ }_{f} T$ and by $[4,2.5], \bar{T}=Q$.

The following is from $[4, \S 5]$ :

Definition 2.12. (i) We define the equivalence relation of "dependency" on $X_{T}$ as follows: If $\chi$ is archimedean, then $\chi \sim \chi^{\prime}$ when $\chi=\chi^{\prime}$. Otherwise $\chi \sim \chi^{\prime}$ when $A(\chi) A\left(\chi^{\prime}\right) \neq K$.

(ii) We set $A[\chi]=\prod_{\chi^{\prime} \sim \chi} A\left(\chi^{\prime}\right)$, clearly a real valuation ring, and we set $T[\chi]=\left(\cap_{\chi^{\prime} \sim \chi} \operatorname{ker} \chi^{\prime}\right) \cup\{0\}$, clearly a preorder of finite exponent. If $X_{T}$ has only 1 dependency class, we write $A_{T}$ for $A[\chi]$ ( $\chi$ any element of $\left.X_{T}\right)$.

Becker and Rosenberg prove results on $A[\chi]$ and $T[\chi]$ when $T$ is of finite index. However, their proofs do not use the full strength of this assumption and thus more general statements hold. 
LEMMA 2.13 (cf. [4, 5.6 and 5.7]). Let $K$ be a field and $T$ a preorder such that $X_{T}$ has only finitely many dependency classes $\left[\chi_{1}\right], \ldots,\left[\chi_{s}\right]$. We write $T_{i}=T\left[\chi_{i}\right]$. Further suppose that for all non-archimedean $\chi_{i}, A\left[\chi_{i}\right] \neq$ K. Then

(i) $X_{T_{i}}=\left[\chi_{i}\right]$

(ii) The natural map $\dot{K} / \dot{T} \rightarrow \Pi_{1}^{s} \dot{K} / \dot{T}_{i}$ is an isomorphism, and hence $\left(X_{T}, \dot{K} / \dot{T}\right)=\oplus_{i=1}^{s}\left(X_{T_{i}}, \dot{K} / \dot{T}_{i}\right)$.

Proof. If $\chi_{i}$ is archimedean, (i) is clear. For non-archimedean $\chi_{i}$, the proof of (i) is exactly that of $[4,5.6(i)]$. The proof of (ii) is exactly that of $[4,5.7]$.

Proof of Theorem 2.8. Let $\alpha_{i}$ be the transcendence degree of $K_{i}$ over Q. We first show that w.1.o.g we can assume $\alpha_{1}=\alpha_{2}$. Suppose $\alpha_{1}<\alpha_{2}$.

Let $v$ be the $x$-adic valuation on $K_{1}(x)$, then $k_{v}=K_{1}$ and $\Gamma_{v}=\mathbf{Z}$. Let $n=$ level $T_{1}$ and let $\Gamma^{\prime}=\left\{k /(2 n)^{m}: k, m \in \mathbf{Z}\right\}$

By $[9,28.1]$, there is an extension of valued fields $(L, w) \supseteq\left(K_{1}(x), v\right)$ such that $L$ is algebraic over $K_{1}(x), \Gamma_{\omega}=\Gamma^{\prime}$ and $k_{\omega}=K_{1}$. In particular, $\Gamma_{\omega}$ is $2 n$-divisible.

Let $\left(K, v^{\prime}\right)$ be a henselization of $(L, w)$. By [2.11], there is a preorder $T$ of $K$ such that $\bar{T}=T_{1}$. Then, by [2.10], $\left(X_{T}, \dot{K} / \dot{T}\right)=\left(X_{T_{1}}, \dot{K}_{1} / \dot{T}_{1}\right)$ and clearly the transcendence degree of $K$ over $\mathbf{Q}$ is $\alpha_{1}+1$.

Using Zorn's lemma and the above construction, we see that we can replace $K_{1}$ with a field of transcendence degree $\alpha_{2}$ over $\mathbf{Q}$ without changing the SOS. Thus w.l.o.g. we can assume $\alpha_{1}=\alpha_{2}$.

Let $L$ be a purely transcendental extension of $\mathbf{Q}$ of degree $\alpha_{1}$, then $K_{1}$ and $K_{2}$ are algebraic over $L$. Let $v$ be the $x$-adic valuation on $L(x)$ and let $\omega$ be the degree valuation on $L(x)$. As above, by $[9,28.1]$ there is an algebraic extension of $L(x), L^{\prime}$, and valuations $v^{\prime}$ and $w^{\prime}$ extending $v$ and $w$ such that $k_{v^{\prime}}=K_{1}, k_{w^{\prime}}=K_{2}, \Gamma_{v^{\prime}}$ is $2 n_{1}$-divisible and $\Gamma_{w^{\prime}}$ is $2 n_{2}$-divisible, where $n_{i}=$ level $T_{i}$.

Let $\left(M_{1}, v^{\prime \prime}\right)$ be a henselization of $\left(L^{\prime}, v^{\prime}\right)$ and let $\left(M_{2}, w^{\prime \prime}\right)$ be a henselization of $\left(L^{\prime}, w^{\prime}\right)$, both in the same algebraic closure of $L^{\prime}$. By [2.11], there are preorders $Q_{i}$ of $M_{i}$ such that $\bar{Q}_{i}=T_{i}$. Hence, by [2.10], $\left(X_{T_{i}}, \dot{M}_{i} / \dot{Q}_{i}\right)=\left(X_{T_{i}}, \dot{K}_{i} / \dot{T}_{i}\right)$

Now we let $F=M_{1} \cap M_{2}$ and $T=Q_{1} \cap Q_{2}$. Set $v_{0}=\left.v^{\prime \prime}\right|_{F}$ and $w_{0}=\left.w^{\prime \prime}\right|_{F}$. Since $L^{\prime} \subseteq F, M_{1}$ and $M_{2}$ are algebraic over $F$ and thus, since $v$ and $w$ are independent, $v_{0}$ and $\omega_{0}$ are independent. Also note that $\left(M_{1}, v^{\prime \prime}\right)$ is a henselization of $\left(F, v_{0}\right)$ and $\left(M_{2}, w^{\prime \prime}\right)$ is a henselization of $\left(F, w_{0}\right)$. In particular, $\left(M_{1}, v^{\prime \prime}\right) \supseteq\left(F, v_{0}\right)$ and $\left(M_{2}, w^{\prime \prime}\right) \supseteq\left(F, w_{0}\right)$ are 
immediate extensions and thus, by [2.9], the diagonal map $\dot{F} / \dot{T} \rightarrow \dot{M}_{1} / \dot{Q}_{1}$ $\times \dot{M}_{2} / \dot{Q}_{2}$ is an isomorphism.

It remains to show that $X_{T}=X_{Q_{1}} \sqcup X_{Q_{2}}$. First we note that $F, M_{1}$ and $M_{2}$ have no archimedean orders: If $P$ were an archimedean order on $M_{1}$, then since $v^{\prime \prime} \sim P[2.11]$, we would have $M_{1}=A(P) \subseteq A_{v^{\prime \prime}}[3,2.7]$, a contradiction. Similarly, $M_{2}$ has no archimedean orders. By $[19,1.3]$ and [1, Chap. 1, Lemma 6], any archimedean order on $F$ would lift to one on $M_{1}$ or $M_{2}$, thus $F$ has no archimedean orders.

Now given $\chi \in X_{T}$, we claim that $v_{0} \sim \chi$ or $w_{0} \sim \chi$. Let $A_{1}$ be the valuation ring of $v_{0}$ and let $A_{2}$ be the valuation ring of $w_{0}$. First suppose that $A(\chi), A_{1}$ and $A_{2}$ are pairwise independent. Let $\left(M_{3}, v_{1}\right)$ be a henselization of $(F, A(\chi))$, then by [2.11] there is a preorder $T_{3}$ of $M_{3}$ such that $\bar{T}_{3}=\operatorname{ker} \chi$. By [2.9] the diagonal map $\dot{F} \rightarrow \dot{M}_{1} / \dot{Q}_{1} \times \dot{M}_{2} / \dot{Q}_{2} \times$ $\dot{M}_{3} / \dot{T}_{3}$ is onto, which is a contradiction since $\dot{F} \rightarrow \dot{M}_{1} / \dot{Q}_{1} \times \dot{M}_{2} / \dot{Q}_{2}$ is onto. Thus, since $A_{1}$ and $A_{2}$ are independent, either $A(\chi) A_{1} \neq F$ or $A(\chi) A_{2} \neq F$, say $A(\chi) A_{1} \neq F$. By our construction of $\Gamma_{v_{0}}, v_{0}$ is a rank 1 valuation. Then, since $A_{1} \subseteq A(\chi) A_{1}$ we must have $A_{1}=A(\chi) A_{1}$ and so $A(\chi) \subseteq A_{1}$. Thus $v_{0} \sim \chi$. Hence any $\chi \in X_{T}$ has $v_{0} \sim \chi$ or $w_{0} \sim \chi$.

Fix $\chi_{i}^{\prime} \in X_{Q_{i}}$ and let $\chi_{i}=\left.\chi_{i}^{\prime}\right|_{F}$. Then clearly $v_{0} \sim \chi_{1}$ and $w_{0} \sim \chi_{2}$. Suppose $\chi_{1}$ and $\chi_{2}$ are in the same dependency class in $X_{T}$, then $A\left(\chi_{1}\right) A\left(\chi_{2}\right)=A \neq F$. Since valuation rings containing a given one are linearly ordered [5, Chap. 6, 4.1], either $A \subseteq A_{1}$ or $A_{1} \subseteq A$.

As above, we must have $A \subseteq A_{1}$, and similarly $A \subseteq A_{2}$. But this implies that $A_{1}$ and $A_{2}$ are linearly ordered which is impossible since they are independent. Thus $\left[\chi_{1}\right] \neq\left[\chi_{2}\right]$.

Since every $\chi \in X_{T}$ has $v_{0} \sim \chi$ or $w_{0} \sim \chi$, every $\chi$ is in $\left[\chi_{1}\right]$ or $\left[\chi_{2}\right]$. Furthermore, $A\left[\chi_{1}\right] \subseteq A_{1}$ and $A\left[\chi_{2}\right] \subseteq A_{2}$. Let $S_{i}=T\left[\chi_{i}\right]$, then, by [2.13], the diagonal map $\dot{F} / \dot{T} \rightarrow \dot{F} / \dot{S}_{1} \times \dot{F} / \dot{S}_{2}$ is an isomorphism and $X_{T}=X_{S_{1}} \sqcup X_{S_{2}}$.

We claim that $S_{i}=Q_{i} \cap F$. If $\chi\left(Q_{1} \cap F\right)=1$, then $v_{0} \sim \chi$ and so $\chi \in X_{S_{1}}$. Hence $X_{Q_{1} \cap F} \subseteq X_{S_{1}}$ and thus $S_{1} \subseteq Q_{1} \cap F[4,1.4]$. Similarly, $S_{2} \subseteq Q_{2} \cap F$. Given $x \in Q_{1} \cap F$. By the above, there is a $y \dot{T} \in \dot{F} / \dot{T}$ which maps to $\left(x \dot{S}_{1}, \dot{S}_{2}\right)$ under the diagonal map. Now, $y \in S_{2} \subseteq Q_{2}$ and $y x^{-1} \in S_{1} \subseteq Q_{1}$, thus $y \in Q_{1} \cap Q_{2}=T$, since $x \in Q_{1}$, and thus $x \in S_{1}$. Hence $Q_{1} \cap F \subseteq S_{1}$ and similarly we see that $Q_{2} \cap F \subseteq S_{2}$. Thus $S_{i}=$ $Q_{i} \cap F$.

Given $\chi \in X_{T}, \quad \chi\left(Q_{1} \cap F\right)=1$ or $\chi\left(Q_{2} \cap F\right)=1$. Suppose $\chi\left(Q_{1} \cap F\right)=1$, then since the diagonal map $\dot{F} / \dot{T} \rightarrow \dot{M}_{1} / \dot{Q}_{1} \times \dot{M}_{2} / \dot{Q}_{2}$ is an isomorphism, $\chi$ lifts to a character $\theta \in\left(\dot{M}_{1} / \dot{Q}_{1}\right)^{*}$. An easy check shows that $\bar{\chi}=\bar{\theta}$ in the residue class field of $v_{0}$ and $v^{\prime \prime}$ and hence 
$\theta \in X_{Q_{1}}[3,2.7]$. Similarly, if $\chi\left(Q_{2} \cap F\right)=1, \chi$ lifts to $X_{Q_{2}}$. Thus $X_{T}=X_{Q_{1}} \sqcup X_{Q_{2}}$ and we are done.

\section{Finitely constructible spaces of signatures and the reduced Witt} ring.

DeFINITION 3.1. We define $m$-constructible SOS's inductively as follows: $(X, G)$ is 1 -constructible iff $(X, G)=\mathscr{C}_{2}$. For $m>1,(X, G)$ is $m$-constructible iff one of the following holds:

(a) There exist SOS's $\left(X_{1}, G_{1}\right)$ and $\left(X_{2}, G_{2}\right)$ and $k<m$ such that each $\left(X_{i}, G_{i}\right)$ is $k$-constructible and $(X, G)=\left(X_{1}, G_{1}\right) \oplus\left(X_{2}, G_{2}\right)$.

(b) There exists a SOS $\left(X_{0}, G_{0}\right)$ and $k<m$ such that $\left(X_{0}, G_{0}\right)$ is $k$-constructible and $(X, G)$ is a group extension of $\left(X_{0}, G_{0}\right)$.

(c) $(X, G)$ is $k$-constructible for some $k<m$.

We say $(X, G)$ is finitely constructible if it is $m$-constructible for some $m \in \mathbf{N}$.

Definition and Remark 3.2 [4, Sec. 5]. The pushdown of a signature $\chi$ along $A(\chi)$ is archimedean and thus induces a unique order embedding of $k(\chi)$ into $\mathbf{R}$. Thus $\chi$ leads to a real-valued place $\lambda(\chi)$ with valuation ring $A(\chi)$. Set $M_{T}=\left\{\lambda(\chi): \chi \in X_{T}\right\}$.

If $\lambda(\chi)=\lambda\left(\chi^{\prime}\right)$, then $A(\chi)=A\left(\chi^{\prime}\right)$ and thus if $\chi$ is non-archimedean, $\chi \sim \chi^{\prime}$ [2.12]. If $\chi$ is archimedean, then $\lambda(\chi)=\lambda\left(\chi^{\prime}\right)$ iff $\chi=\chi^{\prime}$ [5, Prop. 5, Sec. 3, No. 2]. Hence the number of dependency classes of $X_{T}$ is $\leq\left|M_{T}\right|$.

LEMMA 3.3. If $\left|M_{T}\right|=1$, then $T$ is a fan.

Proof. Let $A=A(\chi)$ for some $\chi \in X_{T}$. By assumption, $A\left(\chi^{\prime}\right)=A$ for all $\chi^{\prime} \in X_{T}$. Thus $A \sim{ }_{f} T$ and $\bar{T}$, the pushdown along $A$, is a fan. Hence, by [2.4], $T$ is a fan.

LEMMA 3.4. Given a valuation ring $v$ such that $v \sim{ }_{f} T$. Then $\left|M_{\bar{T}}\right|=$ $\left|M_{T}\right|$

Proof. Given $\lambda \in M_{T}$, define $\theta(\lambda) \in M_{\bar{T}}$ via $\theta(\lambda)(u+I)=\lambda(u)$. Given $\lambda^{\prime} \in M_{\bar{T}}$, define $\theta^{\prime}\left(\lambda^{\prime}\right) \in M_{T}$ via $\theta^{\prime}\left(\lambda^{\prime}\right)(x)=\infty$ if $x \notin A$ and $\theta^{\prime}\left(\lambda^{\prime}\right)(x)=\lambda^{\prime}(x+I)$ for $x \in A$. Then clearly $\theta\left(\theta^{\prime}\right)=$ identity on $M_{\bar{T}}$ and $\theta^{\prime}(\theta)=$ identity on $M_{T}$. Thus $\theta$ is a bijection and so $\left|M_{\bar{T}}\right|=\left|M_{T}\right|$.

We are now ready to prove our main theorem. 
THEOREM 3.5. $(X, G)$ is finitely constructible iff $(X, G)$ is realizable by a field $K$ and a preorder $T$ such that $\left|M_{T}\right|<\infty$.

Proof. If $(X, G)$ is $m$-constructible we use induction on $m$ and [2.5] and [2.8] to show that $(X, G)$ is realizable. Note that if $X_{T}=X_{T_{1}} \sqcup X_{T_{2}}$, then clearly $\left|M_{T}\right| \leq\left|M_{T_{1}}\right|+\left|M_{T_{2}}\right|$ and thus direct sum preserves finiteness of $M_{T}$. By [3.4], our group extension construction does also and hence the preorder $T$ constructed has $\left|M_{T}\right|<\infty$.

Now suppose that $T$ is a preorder with $\left|M_{T}\right|<\infty$. We induct on $\left|M_{T}\right|$. If $\left|M_{T}\right|=1$ we are done by [3.3], since every fan is $\mathscr{C}_{2}$ or a group extension of $\mathscr{C}_{2}$.

Suppose $\left|M_{T}\right|>1$. By [3.2], $X_{T}$ has only finitely many dependency classes.

Case 1. The dependency classes of $X_{T}$ are $X_{T_{1}}, \ldots, X_{T_{s}}$ where $s>1$. By [3.2] we have $M_{T}=M_{T_{1}} \sqcup \cdots \sqcup M_{T_{s}}$ and thus by induction each $\left(X_{T_{i}}, \dot{K} / \dot{T}_{i}\right)$ is finitely constructible. Hence, by $\left[2.13\right.$, ii] $\left(X_{T}, \dot{K} / \dot{T}\right)$ is finitely constructible.

Case 2. Suppose $X_{T}$ has 1 dependency class. Since $\left|M_{T}\right|>1, T$ is non-archimedean. Since $\left|M_{T}\right|<\infty$, there are only finitely many $A(\chi)$ 's, $\chi \in X_{T}$, and thus, as in $[4,5.6(\mathrm{i})]$, we see that $A_{T} \neq K$. Let $\bar{T}$ be the pushdown of $T$ along $A_{T}$, then by [2.3] it suffices to show that $\left(X_{\bar{T}}, \dot{k} / \dot{\bar{T}}\right)$ is finitely constructible.

If $X_{\bar{T}}$ has more than one dependency class then, since $\left|M_{\bar{T}}\right|=\left|M_{T}\right|$ [3.4], the proof of Case 1 shows that $\left(X_{\bar{T}}, \dot{k} / \dot{\bar{T}}\right)$ is finitely constructible. If $X_{\bar{T}}$ has 1 dependency class, then $A_{\bar{T}}=k$ [2, p. 1961-1963]. As above, this implies that $\bar{T}$ is archimedean. In particular, $\bar{T}$ is a fan and we are done.

Definition 3.6. (i) Let $(X, G)$ be a SOS. For a form $f$ over $(X, G)$ denote by [f] its $X$-equivalence class [1.1]. The set $W(X)$ of equivalence classes of forms over $(X, G)$ carries a natural ring structure as follows: We define $[f]+[g]$ to be $[f \oplus g]$ and we define $[f] \cdot[g]$ to be $[f \otimes g]$. Then if we define $-[f]$ to be $[-f]$, clearly $(W(X),+, \cdot)$ is a commutative ring with $1=[\langle 1\rangle]$ and $0=[\langle 1,-1\rangle]$, the class of all hyperbolic forms. The ring $W(X)$ is called the Witt Ring of $(X, G)$.

(ii) Let $I(X)$ in $W(X)$ be the set of classes of even-dimensional forms. That this is well defined follows from the fact that if $f$ and $g$ are $X$-equivalent forms, then $\operatorname{dim} f \equiv \operatorname{dim} g(\bmod 2)$, which can be shown as in the proof of $[4,4.8]$. It is clear that $I(X)$ is an ideal. 
REMARK 3.7. Given a field $K$ and preorder $T$ then $W_{T}$, the higher level reduced Witt ring of $[4,4.2]$, is easily seen to be isomorphic to $W\left(X_{T}\right)$.

Definition 3.8 [18, p. 13]. (i) Given a commutative ring $R$, denote by $U(R)$ the abelian group of units of $R$.

(ii) Given a commutative ring $R$ and an abelian group $H$. Suppose $\lambda$ : $H \times H \rightarrow U(R)$ is a factor set. We define the twisted group ring of $R$ over $H$ by $\lambda, R^{t}[H]$, as the set of formal sums $\sum_{h \in H}\left(r_{h}, h\right)$ where $r_{h} \in R$ and only finitely many $r_{h}$ 's are non-zero. If $\sum_{h \in H}\left(s_{h}, h\right)$ is another element of $R^{t}[H]$ then addition is defined by $\Sigma\left(r_{h}, h\right)+\Sigma\left(s_{h}, h\right)=\Sigma\left(r_{h}+s_{h}, h\right)$, and multiplication is defined by $\left(\Sigma\left(r_{h}, h\right)\right) \cdot\left(\Sigma\left(s_{h}, h\right)\right)=\Sigma\left(z_{h}, h\right)$, where $z_{h}=\sum_{h_{1} h_{2}=h} \lambda\left(h_{1}, h_{2}\right) r_{h_{1}} s_{h_{2}}$. Then $R^{t}[H]$ is a commutative ring with $0=$ $\left(0_{R}, 1_{H}\right)$ and $1=\left(1_{R}, 1_{H}\right)$.

Definition 3.9 (cf. [4, 2.10] and [17, 3.11]). Given a SOS $(X, G)$ which is a group extension of $\left(X_{0}, G_{0}\right)$. Then we have an exact sequence $1 \rightarrow G_{0} \rightarrow G \rightarrow G / G_{0} \rightarrow 1$ which gives a rise to a factor set $\lambda: G / G_{0} \times$ $G / G_{0} \rightarrow G_{0}$ such that under this bijection the multiplication in $G$ is given by: $\left(e_{1}, h_{1}\right) \cdot\left(e_{2}, h_{2}\right)=\left(\lambda\left(h_{1}, h_{2}\right) e_{1} e_{2}, h_{1} h_{2}\right)$ for all $e_{i} \in G_{0}$ and $h_{i} \in$ $G / G_{0}$.

Given $f=\left\langle a_{1}, \ldots, a_{n}\right\rangle$, a form over $\left(X_{0}, G_{0}\right)$, and $h \in G / G_{0}$, let $\{f, h\}=\left\langle\left(a_{1}, h\right), \ldots,\left(a_{n}, h\right)\right\rangle$, a form over $(X, G)$. Let $f$ be any form over $(X, G)$, then for each $h \in G / G_{0}$ there is obviously a (possibly empty) form $f_{h}$ over $\left(X_{0}, G_{0}\right)$, called the hth residue form of $f$, such that $f=$ $\bigoplus_{h \in G / G_{0}}\left\{f_{h}, h\right\}$.

LEMMA 3.10 (cf. [4, 4.7]). Let $f$ and $g$ be forms over $(X, G)$. Then $[f]=[g]$ in $W(X)$ iff $\left[f_{h}\right]=\left[g_{h}\right]$ in $W\left(X_{0}\right)$ for all $h \in G / G_{0}$.

Proof. This follows easily from [17, 2.7].

Proposition 3.11. Given $(X, G)$ a group extension of $\left(X_{0}, G_{0}\right)$. Let $\lambda$ be a factor set arising from the exact sequence $1 \rightarrow G_{0} \rightarrow G \rightarrow G / G_{0} \rightarrow 1$. Then $W(X) \cong W\left(X_{0}\right)^{t}\left[G / G_{0}\right]$, the twisted group ring of $W\left(X_{0}\right)$ by $G / G_{0}$ arising from $\lambda$.

Proof. Define $\varphi: W(X) \rightarrow W\left(X_{0}\right)^{t}\left[G / G_{0}\right]$ by

$$
\varphi([f])=\sum_{h \in G / G_{0}}\left\{\left[\tilde{f}_{h}\right], h\right\},
$$


where $\bar{f}_{h}$ is defined as in [3.9] using $\lambda$. By [3.10], $\varphi$ is well-defined and injective.

A straightforward calculation shows that for $a_{1}, a_{2} \in G, \varphi\left(\left[\left\langle a_{1}\right\rangle\right]+\right.$ $\left.\left[\left\langle a_{2}\right\rangle\right]\right)=\varphi\left(\left[\left\langle a_{1}\right\rangle\right]\right)+\varphi\left(\left[\left\langle a_{2}\right\rangle\right]\right)$. Then, since any $[f]$ is a sum of $[\langle a\rangle]$ 's, it follows easily that $\varphi$ is a homomorphism.

Given a form $\bar{f}=\left\langle e_{1}, \ldots, e_{k}\right\rangle$ over $\left(X_{0}, G_{0}\right)$ and $h \in G / G_{0}$. Let $f$ be the form $\left\langle\left(e_{1}, h\right), \ldots,\left(e_{k}, h\right)\right\rangle$ over $(X, G)$, then clearly $\varphi([f])=\{[\bar{f}], h\}$. Thus $\varphi$ is surjective and therefore $\varphi$ is an isomorphism.

The following is implicit in $[4, \S 2]$ :

COROLlary 3.12. Given a field $K$, a preorder $T$, and $v$ a valuation such that $v \sim{ }_{f} T$. Then $W\left(X_{T}\right) \cong W\left(X_{\bar{T}}\right)^{t}[\Gamma / v(\dot{T})]$, where the twisting arises from a factor set of the canonical exact sequence $1 \rightarrow \dot{k} / \dot{\bar{T}} \rightarrow \dot{K} / \dot{T} \rightarrow$ $\Gamma / v(\dot{\bar{T}}) \rightarrow 1$.

Proposition 3.13. Given SOS's $(X, G),\left(X_{1}, G_{1}\right)$ and $\left(X_{2}, G_{2}\right)$ such that $(X, G)=\left(X_{1}, G_{1}\right) \oplus\left(X_{2}, G_{2}\right)$. Then $W(X) \cong \mathbf{Z}+\left(I\left(X_{1}\right) \times I\left(X_{2}\right)\right)$ $\subseteq W\left(X_{1}\right) \times W\left(X_{2}\right)$, where $\mathbf{Z}$ has the diagonal embedding in $W\left(X_{1}\right) \times$ $W\left(X_{2}\right)$.

Proof. Given $f$, a form over $(X, G)$, then $f=\left\langle\left(a_{1}, b_{1}\right), \ldots,\left(a_{m}, b_{m}\right)\right\rangle$, where each $a_{i}$ is in $G_{1}$ and each $b_{i}$ is in $G_{2}$. Thus we write $f=f_{1} \sqcup f_{2}$ where $f_{1}=\left\langle a_{1}, \ldots, a_{m}\right\rangle$, a form over $\left(X_{1}, G_{1}\right)$ and $f_{2}=\left\langle b_{1}, \ldots, b_{m}\right\rangle$, a form over $\left(X_{2}, G_{2}\right)$. Then it follows from the definition of direct sum that $\left[f_{1} \sqcup f_{2}\right]=\left[g_{1} \sqcup g_{2}\right]$ iff $\sigma\left(f_{1} \sqcup f_{2}\right)=\sigma\left(g_{1} \sqcup g_{2}\right)$ for all $\sigma$ in $X$ iff $\sigma_{1}\left(f_{1}\right)$ $=\sigma_{1}\left(g_{1}\right)$ for all $\sigma_{1}$ in $X_{1}$ and $\sigma_{2}\left(f_{2}\right)=\sigma_{2}\left(g_{2}\right)$ for all $\sigma_{2}$ in $X_{2}$ iff $\left[f_{1}\right]=\left[g_{1}\right]$ in $W\left(X_{1}\right)$ and $\left[f_{2}\right]=\left[g_{2}\right]$ in $W\left(X_{2}\right)$. Now, define $\beta: W(X) \rightarrow$ $W\left(X_{1}\right) \times W\left(X_{2}\right)$ by

$$
\beta\left(\left[f_{1} \sqcup f_{2}\right]\right)=\left(\left[f_{1}\right],\left[f_{2}\right]\right) .
$$

By the above, $\beta$ is well-defined and injective.

Now we want to show that the image of $\beta$ is $\mathbf{Z}+I\left(X_{1}\right) \times I\left(X_{2}\right)$. Given $f$, a form over $(X, G)$, when we write $f$ as $f_{1} \sqcup f_{2}$ we have $\operatorname{dimension}\left(f_{1}\right)=\operatorname{dimension}\left(f_{2}\right)$. If $\operatorname{dim}\left(f_{i}\right)$ is even, then $\beta\left(\left[f_{1} \sqcup f_{2}\right]\right)=$ $\left(\left[f_{1}\right],\left[f_{2}\right]\right) \subseteq I\left(X_{1}\right) \times I\left(X_{2}\right)$. If $\operatorname{dim}\left(f_{l}\right)$ is odd, then

$$
\begin{aligned}
\beta\left(\left[f_{1} \sqcup f_{2}\right]\right) & =\left(\left[f_{1}\right],\left[f_{2}\right]\right)=\left(\left[\langle 1,-1\rangle \oplus f_{1}\right],\left[\langle 1,-1\rangle \oplus f_{2}\right]\right) \\
& =1+\left(\left[\langle-1\rangle \oplus f_{1}\right],\left[\langle-1\rangle \oplus f_{2}\right]\right) \subseteq \mathbf{Z}+I\left(X_{1}\right) \times I\left(X_{2}\right) .
\end{aligned}
$$

Thus Image $(\beta) \subseteq \mathbf{Z}+I\left(X_{1}\right) \times I\left(X_{2}\right)$. 
Given $n+\left(\left[g_{1}\right],\left[g_{2}\right]\right)$ in $\mathbf{Z}+I\left(X_{1}\right) \times I\left(X_{2}\right)$. Suppose $g_{1}=$ $\left\langle a_{1}, \ldots, a_{2 m}\right\rangle$ and $g_{2}=\left\langle b_{1}, \ldots, b_{2 k}\right\rangle$, then w.1.o.g. we can assume $m \leq k$. Let

$$
\begin{array}{r}
f=n\langle 1\rangle \oplus\left\langle\left(a_{1}, b_{1}\right), \ldots,\left(a_{2 m}, b_{2 m}\right),\left(1, b_{2 m+1}\right),\right. \\
\left.\left(-1, b_{2 m+2}\right), \ldots,\left(-1, b_{2 k}\right)\right\rangle,
\end{array}
$$

a form over $(X, G)$. Then $\beta(f)=n+\left(\left[g_{1} \oplus(m-k)\langle 1,-1\rangle\right],\left[g_{2}\right]\right)=$ $n+\left(\left[g_{1}\right],\left[g_{2}\right]\right)$. Hence $\mathbf{Z}+I\left(X_{1}\right) \times I\left(X_{2}\right) \subseteq \operatorname{Image}(\beta)$ and we are done.

Definition 3.14. A ring $R$ is finitely realizable if there is a field $K$ and a preorder $T$ with $\left|M_{T}\right|<\infty$ such that $W\left(X_{T}\right) \cong R$.

Using our previous result on finitely constructible SOS's [3.5], we can now give an inductive construction for finitely realizable rings. In the case where $G^{2}=1$ this is due to Craven.

THEOREM 3.15 (cf. $[8,2.1])$. Finitely realizable rings are precisely those given by the following inductive construction:

(a) $\mathbf{Z}$ is finitely realizable.

(b) If $R_{1}$ and $R_{2}$ are finitely realizable, then $R=\mathbf{Z}+I_{1} \times I_{2}$ is also, where $I_{1}$ and $I_{2}$ are the ideals of even dimensional forms.

(c) If $R_{0}$ is finitely realizable, $H$ is abelian of finite exponent and $\lambda$ : $H \times H \rightarrow U(R)$ is a factor set with image $(\lambda) \subseteq\left\{r \in U\left(R_{0}\right): r\right.$ has finite exponent $\}$, then $R_{0}^{t}[H]$ is finitely realizable, where the twisting arises from $\lambda$.

Proof. This follows easily from [3.5], using [3.11], [3.13] and the fact that for a preordered field $(K, T), \dot{K} / \dot{T}=\{r \in U(R): r$ has finite exponent\} [Becker, Rosenberg, unpublished].

We now wish to make use of [3.5] to study the number of signatures in $X_{T}$ for a preorder $T$ of finite index. In the case where $G^{2}=1$, this was done by Bröcker [6].

Definition 3.16. (i) Let $A$ be the semigroup $\oplus_{i=1}^{\infty} \mathbf{Z}^{+}$with addition defined in the usual way. For $\alpha=\left(e_{1}, \ldots, e_{m}, 0, \ldots\right)$ in $A$, (all entries beyond the $m$ th one are 0 ) and $p$ a prime number, let $G_{\alpha, p}=e_{1} C_{p} \times e_{2} C_{p^{2}}$ $\times \cdots \times e_{m} C_{p^{m}}$, where $C_{n}=\mathbf{Z} / n \mathbf{Z}$ and $k C_{n}=C_{n} \times C_{n} \times \cdots \times C_{n}, k$ times. Set $B=\bigoplus_{j=1}^{\infty} A$.

(ii) For $\bar{\alpha}=\left(\alpha_{1}, \ldots, \alpha_{k}, 0, \ldots\right)$ in $B$ (each $\alpha_{i}$ is in $A$ and all entries beyond the $k$ th one are 0 ), set $G_{\bar{\alpha}}=G_{\alpha_{1}, p_{1}} \times G_{\alpha_{2}, p_{2}} \times \cdots \times G_{\alpha_{k}, p_{k}}$, where $\left\{p_{1}<p_{2}<p_{3}<\cdots\right\}$ is the set of prime numbers. 
(iii) Given $\bar{\alpha}, \bar{\beta}$ and $\bar{\gamma}$ in $B,(\bar{\alpha}, \bar{\beta}) \sim \bar{\gamma}$ if there is an exact sequence $1 \rightarrow G_{\bar{\gamma}} \rightarrow G_{\bar{\alpha}} \rightarrow G_{\bar{\beta}} \rightarrow 1$.

(iv) For $\alpha=\left(e_{1}, e_{2}, \ldots, e_{m}, 0,0, \ldots\right)$ in $A$, define $|\alpha|=\sum_{j=1}^{m} j \cdot e_{j}$. Each part of the following lemma is clear or a direct consequence of the Fundamental Theorem for abelian groups.

LEMMA 3.17. (i) If $\alpha=\left(\alpha_{1}, \ldots, \alpha_{k}, 0,0, \ldots\right)$ is in $B$, then $\left|G_{\bar{\alpha}}\right|=$ $p_{1}^{\left|\alpha_{1}\right|} \cdot p_{2}^{\left|\alpha_{2}\right|} \cdots \cdot p_{k}^{\left|\alpha_{k}\right|}$.

(ii) $G$ is a finite abelian group iff $G=G_{\bar{\alpha}}$ for some $\bar{\alpha}$ in $B$.

(iii) $\bar{\gamma}=\bar{\alpha}+\bar{\beta}$ iff $G_{\bar{\alpha}} \times G_{\bar{\beta}} \cong G_{\bar{\gamma}}$. Thus if $\bar{\gamma}=\bar{\alpha}+\bar{\beta}$, then $(\bar{\alpha}, \bar{\beta}) \sim \bar{\gamma}$.

Definition 3.18. For $\bar{\alpha}$ in $B$, we define $S(\bar{\alpha})$, a subset of $\mathbf{N}$, recursively as follows:

$$
S(((1,0,0, \ldots), 0,0, \ldots))=\{1\} .
$$

For all other $\bar{\alpha}, a \in S(\bar{\alpha})$ iff there are $\bar{\alpha}_{1}$ and $\bar{\alpha}_{2}$ in $B$ such that:

1. $\bar{\alpha}=\bar{\alpha}_{1}+\bar{\alpha}_{2}$ and $a=a_{1}+a_{2}$ for some $a_{i} \in S\left(\bar{\alpha}_{i}\right)$ or

2. $\left(\bar{\alpha}, \bar{\alpha}_{2}\right) \sim \bar{\alpha}$ and $a=\left|G_{\bar{\alpha}_{2}}\right| \cdot a_{1}$ for some $a_{1} \in S\left(\bar{\alpha}_{1}\right)$.

Note that pairs $\left(\bar{\alpha}_{1} \cdot \bar{\alpha}_{2}\right)$ with $\left(\bar{\alpha}_{1} \cdot \bar{\alpha}_{2}\right) \sim \bar{\alpha}$ correspond to abelian group extensions of $G_{\bar{\alpha}_{2}}$ by $G_{\bar{\alpha}_{1}}$. Since $\operatorname{Ext}\left(H_{1}, H_{2}\right)$ is finite for finite abelian groups $H_{1}$ and $H_{2}$ [10, p. 222], for a fixed $\bar{\alpha}$ in $B$ there are only finitely many pairs $\left(\bar{\alpha}_{1}, \bar{\alpha}_{2}\right)$ with $\left(\bar{\alpha}_{1}, \bar{\alpha}_{2}\right) \sim \bar{\alpha}$. Thus any $S(\bar{\alpha})$ can be "constructed" in a finite number of steps.

TheOREM 3.19 (cf. [6, 3.21]). Suppose $\bar{\alpha} \in B$ and $K$ is a field with a preorder $T$ such that $\dot{K} / \dot{T} \cong G_{\bar{\alpha}}$, then $\left|X_{T}\right| \in S(\bar{\alpha})$. Conversely, given any $\bar{\alpha} \in B$, then for any $a \in S(\bar{\alpha})$ there is a field $K$ and a preorder $T$ such that $\dot{K} / \dot{T} \cong G_{\bar{\alpha}}$ and $\left|X_{T}\right|=a$.

Proof. For the first statement, we induct on $\left|G_{\bar{\alpha}}\right|$. If $\left|G_{\bar{\alpha}}\right|=2$, then $\bar{\alpha}=((1,0,0, \ldots), 0,0, \ldots)$ and $\left|X_{T}\right|=1$ which is in $S(\bar{\alpha})$.

Suppose $\left|G_{\bar{\alpha}}\right|>2$. Then, by [3.5], $\left(X_{T}, \dot{K} / \dot{T}\right)$ is a direct sum or a group extension. If it is a direct sum, we are done by induction and (1) of the definition of $S(\bar{\alpha})$.

Suppose $\left(X_{T}, \dot{K} / \dot{T}\right)$ is a group extension of $\left(X_{T}, \dot{K}_{1} / \dot{T}_{1}\right)$. Let $H=$ $(\dot{K} / \dot{T}) /\left(\dot{K}_{1} / \dot{T}_{1}\right)$ and say $\dot{K}_{1} / \dot{T}_{1} \cong G_{\bar{\alpha}_{1}}$ and $H \cong G_{\bar{\alpha}_{2}}$ [3.17]. Then, by definition, $\left(\bar{\alpha}_{1} \cdot \bar{\alpha}_{2}\right) \sim \bar{\alpha}$ and by induction $\left|X_{T_{1}}\right| \in S\left(\bar{\alpha}_{1}\right)$. By [2.2, ii], $\left|X_{T}\right|=\left|X_{T_{1}}\right| \cdot\left|G_{\bar{\alpha}_{2}}\right|$ which is in $S(\bar{\alpha})$ by (2) of the definition.

For the second statement we again induct on $\left|G_{\bar{\alpha}}\right|$. For (1) we use [2.8] and for (2) we use [2.5]. 
Acknowledgment. This paper represents a portion of the author's doctoral thesis, written under the supervision of Professor Alex Rosenberg at Cornell University. The author gratefully acknowledges the help and encouragement of Professor Rosenberg.

\section{REFERENCES}

[1] E. Becker, Hereditarily-Pythagorean Fields and Orderings of Higher Level, Lecture Notes 29, Instituto de Mathematica Pura e Aplicado, Rio de Janeiro, 1978.

[2] _ Partial orders on a field and valuation rings, Comm. Algebra, 7 (1979), 1933-1976.

[3] E. Becker, J. Harmon, and A. Rosenberg, Signatures of fields and extension theory, J. Reine Angew. Math., 330 (1978), 53-75.

[4] E. Becker and A. Rosenberg, Reduced forms and reduced Witt rings of higher level, J. Algebra, 92 (1985), 477-503.

[5] N. Bourbaki, Algebré Commutative, Chaps. 5-6, Hermann, Paris, 1964.

[6] L. Bröcker, Über die Anzahl der Anordungen eines kommutativen Körpers, Arch. Math., 29 (1977), 458-463.

[7] H. Cartan and S. Eilenberg, Homological Algebra, Princeton University Press, Princeton, N.J., 1956.

[8] T. Craven, Characterizing reduced Witt rings of fields, J. Algebra, 53 (1978), 68-77.

[9] O. Endler, Valuation theory, Universitext, Springer-Verlag, New York, 1972.

[10] L. Fuchs, Infinite Abelian Groups, Volume 1, Academic Press, New York, 1970.

[11] T. Y. Lam, The Algebraic Theory of Quadratic Forms, Benjamin/Cummings, Reading, Mass., 1973.

[12] - Orderings, Valuations and Quadratic Forms, CBMS Regional Conference Series in Mathematics, Vol. 52, Amer. Math. Soc., Providence, R.I., 1983.

[13] M. Marshall, Classification of finite spaces of orderings, Canad. J. Math., 31 (1978), 320-330.

[14] M. Marshall, Quotients and inverse limits of spaces of orderings, Canad. J. Math., 31 (1979), 604-616.

[15] The Witt ring of a space of orderings, Trans. Amer. Math. Soc., 258 (1980), 505-521.

[16] _. Spaces of Orderings IV, Canad. J. Math., 32 (1980), 603-627.

[17] C. Mulcahy, An abstract approach to higher level forms and rigidity, to appear.

[18] D. Passman, The Algebraic Structure of Group Rings, Wiley, New York, 1977.

[19] A Prestel, Remarks on the Pythagoras and Hasse numbers of real fields, J. Reine Angew. Math., 303 (1978), 284-294.

[20] S. Priess-Crampe, Angeordnete Strukturen: Gruppen, Körper, projektive Ebenen, Ergebnisse der Mathematik und ihrer Grenzgebiete 98, Springer-Verlag, Berlin, 1983.

Received February 17, 1986 and in revised form May 29, 1986.

ThE UNIVERSITY OF HaWAII at MaNOA

HONOLULU, HI 96822 



\title{
PACIFIC JOURNAL OF MATHEMATICS EDITORS
}

\author{
V. S. VARADARAJAN \\ (Managing Editor) \\ University of California \\ Los Angeles, CA 90024 \\ HERBERT Clemens \\ University of Utah \\ Salt Lake City, UT 84112 \\ R. FINN \\ Stanford University \\ Stanford, CA 94305
}

\author{
HERMANN FLASCHKA \\ University of Arizona \\ Tucson, AZ 85721 \\ RAMESH A. GANGOLLI \\ University of Washington \\ Seattle, WA 98195 \\ VAUGHAN F. R. JONES \\ University of California \\ Berkeley, CA 94720 \\ ROBION KIRBY \\ University of California \\ Berkeley, CA 94720
}

\author{
C. C. Moore \\ University of California \\ Berkeley, CA 94720
}

H. SAMELSON

Stanford University

Stanford, CA 94305

HAROLD STARK

University of California, San Diego

La Jolla, CA 92093

\section{ASSOCIATE EDITORS}

\author{
R. ARENS \\ E. F. BECKENBACH \\ B. H. NEUMANN \\ F. WOLF \\ K. YOSHIDA \\ (1906-1982)

\section{SUPPORTING INSTITUTIONS} \\ UNIVERSITY OF ARIZONA \\ UNIVERSITY OF BRITISH COLUMBIA \\ CALIFORNIA INSTITUTE OF TECHNOLOGY \\ UNIVERSITY OF CALIFORNIA \\ MONTANA STATE UNIVERSITY \\ UNIVERSITY OF NEVADA, RENO \\ NEW MEXICO STATE UNIVERSITY \\ OREGON STATE UNIVERSITY

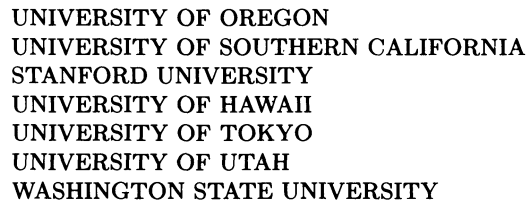

The Supporting Institutions listed above contribute to the cost of publication of this Journal, but they are not owners or publishers and have no responsibility for its content or policies.

Mathematical papers intended for publication in the Pacific Journal of Mathematics should be in typed form or offset-reproduced (not dittoed), double spaced with large margins. Please do not use built up fractions in the text of the manuscript. However, you may use them in the displayed equations. Underline Greek letters in red, German in green, and script in blue. The first paragraph must be capable of being used separately as a synopsis of the entire paper. In particular it should contain no bibliographic references. Please propose a heading for the odd numbered pages of less than 35 characters. Manuscripts, in triplicate, may be sent to any one of the editors. Please classify according to the scheme of Math. Reviews, Index to Vol. 39. Supply name and address of author to whom proofs should be sent. All other communications should be addressed to the managing editor, or Elaine Barth, University of California, Los Angeles, California 90024.

There are page-charges associated with articles appearing in the Pacific Journal of Mathematics. These charges are expected to be paid by the author's University, Government Agency or Company. If the author or authors do not have access to such Institutional support these charges are waived. Single authors will receive 50 free reprints; joint authors will receive a total of $\mathbf{1 0 0}$ free reprints. Additional copies may be obtained at cost in multiples of 50 .

The Pacific Journal of Mathematics is issued monthly as of January 1966. Regular subscription rate: $\$ 190.00$ a year (5 Vols., 10 issues). Special rate: $\$ 95.00$ a year to individual members of supporting institutions.

Subscriptions, orders for numbers issued in the last three calendar years, and changes of address should be sent to Pacific Journal of Mathematics, P.O. Box 969, Carmel Valley, CA 93924, U.S.A. Old back numbers obtainable from Kraus Periodicals Co., Route 100, Millwood, NY 10546.

The Pacific Journal of Mathematics at P.O. Box 969, Carmel Valley, CA 93924 (ISSN 0030-8730) publishes 5 volumes per year. Application to mail at Second-class postage rates is pending at Carmel Valley, California, and additional mailing offices. Postmaster: send address changes to Pacific Journal of Mathematics, P.O. Box 969, Carmel Valley, CA 93924.

PUBLISHED BY PACIFIC JOURNAL OF MATHEMATICS, A NON-PROFIT CORPORATION Copyright (C) 1987 by Pacific Journal of Mathematics 


\section{Pacific Journal of Mathematics}

Vol. 128 , No. $2 \quad$ April, 1987

Pierre Barrucand, John Harold Loxton and Hugh C. Williams, Some

explicit upper bounds on the class number and regulator of a cubic field

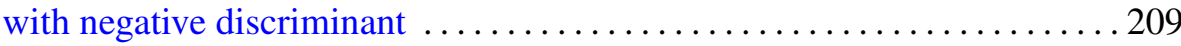

Thomas Ashland Chapman, Piecewise linear fibrations $\ldots \ldots \ldots \ldots \ldots 223$

Yves Félix and Jean-Claude Thomas, Extended Adams-Hilton's

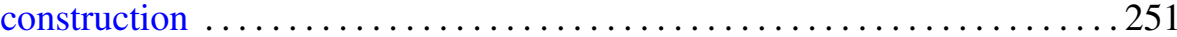

Robert Fitzgerald, Derivation algebras of finitely generated Witt rings . . . 265

K. Gopalsamy, Oscillatory properties of systems of first order linear delay differential inequalities ................................ 299

John P. Holmes, One parameter subsemigroups in locally complete

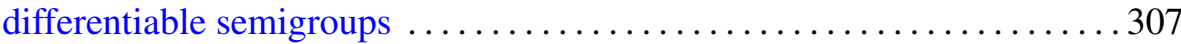

Douglas Murray Pickrell, Decomposition of regular representations for

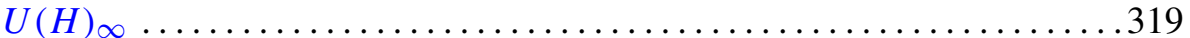

Victoria Powers, Characterizing reduced Witt rings of higher level

Parameswaran Sankaran and Peter Zvengrowski, Stable parallelizability

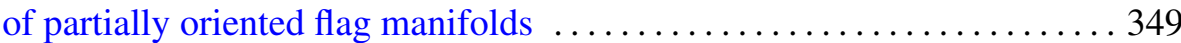

Johan Tysk, Eigenvalue estimates with applications to minimal surfaces . . . 361 Akihito Uchiyama, On McConnell's inequality for functionals of subharmonic functions

Minato Yasuo, Bott maps and the complex projective plane: a construction

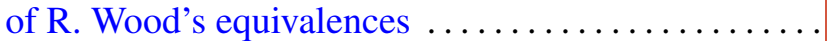

James Juei-Chin Yeh, Uniqueness of strong solutions to stochastic differential equations in the plane with deterministic boundary process 